\title{
Urogonimus turdi (Digenea: Leucochloridiidae) from the White's Thrush, Zoothera aurea, in the Republic of Korea
}

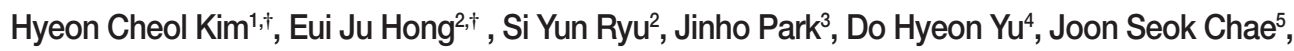 \\ Kyoung Seong $\mathrm{Choi}^{6}$, Cheolho $\mathrm{Sim}^{7}$, Bae Keun Park2,* \\ ${ }^{1}$ College of Veterinary Medicine and Institute of Veterinary Science, Kangwon National University, Chuncheon 24289, Korea; ${ }^{2}$ College of Veterinary \\ Medicine, Chungnam National University, Daejeon 34134, Korea; ${ }^{3}$ College of Veterinary Medicine, Chonbuk National University, Cheongju 28644 , \\ Korea; ${ }^{4}$ College of Veterinary Medicine, Gyeongsang National University, Jinju 52828, Korea; ${ }^{5}$ Laboratory of Veterinary Internal Medicine, BK21 \\ PLUS Program for Creative Veterinary Science Research, Research Institute for Veterinary Science and College of Veterinary Medicine, Seoul \\ National University, Seoul 08826, Korea; ${ }^{6}$ College of Ecology and Environmental Science, Kyungpook National University, Sangju 37224, Korea; \\ ${ }^{7}$ Department of Biology, Baylor University, Waco, Texas 76798, USA
}

\begin{abstract}
Avian trematodes, Urogonimus turdi (Digenea: Leucochloridiidae), were collected from the intestine of wild birds, Zoothera aurea, 2013-2017 in the Daejeon Metropolitan City, Korea. The body was ellipsoidal, attenuated and/or round ends, 1,987-2,120 long and 819-831 $\mu \mathrm{m}$ wide. The oral sucker was subterminal, rounded anteriorly, and 308$425 \times 351-432 \mu \mathrm{m}$ in size; the prepharynx and esophagus were almost lacking; pharynx was well-developed, 142$179 \times 78-170 \mu \mathrm{m}$ in size; intestine narrow, bifurcating just after pharynx, ascending to the oral sucker before looping posteriorly and terminating near the posterior end; ventral sucker larger, in almost median, 536-673×447-605 $\mu \mathrm{m}$ and approximately 1.5 times larger than oral sucker. A phylogenetic tree constructed with 18S ribosomal RNA showed inter- and intraspecific relationships. Based on these morphological and molecular findings, we report here a U. turdi from White's thrushes in Korea.
\end{abstract}

Key words: Urogonimus turdi, Zoothera aurea, White's thrush, Korea

\section{INTRODUCTION}

Genus Urogonimus belongs to family Leucochloridiidae Poche, 1907 comprising 2 other genera, Urotocus Looss, 1899 and Leucochloridium Carus, 1835. Fluke members of the genus Urogonimus are morphologically distinguishable from those of the other 2 genera due to the location of their genital pores [1]. Records of at least 5 species, namely, $U$. cardis, U. caryocatactis, $U$. certhiae, $U$. dryobatae, and $U$. macrostomus have been found in literature. Their validities are still obscure and debatable except $U$. macrostomus. Locke et al. [2] have found molecular and morphological evidences from Holarctic samples that support the validity of $U$. macrostomus.

Trematodes belonging to the Leucochloridiidae were found in many wild birds including passerine and shorebirds [3-6].

- Received 21 October 2018, revised 23 July 2019, accepted 20 August 2019.

*Corresponding author (bkpark@cnu.ac.kr)

${ }^{\dagger}$ This author contributed equally to this work.

(c) 2019, Korean Society for Parasitology and Tropical Medicine

This is an Open Access article distributed under the terms of the Creative Commons Attribution Non-Commercial License (http://creativecommons.org/licenses/by-nc/4.0) which permits unrestricted non-commercial use, distribution, and reproduction in any medium, provided the original work is properly cited.
In Japan, Leucochloridium japonicum was collected from the copper pheasant (Syrmaticus soemmerringii) [6].Other similar species such as L. sime collected from the hawfinch (Coccothraustes coccothraustes) [7], and L. cardis and L. turdi collected from the Japanese thrush (Turdus cardis) [8]. Additionally, $U$. macrostomus was found in the rustic bunting (Emberiza rustica) [9]. Meanwhile, Kagan [10] classified L. cardis and L. turdi into the genus Urogonimus based on the differences in their reproductive organs and the arrangement of their uterine loops, which resulted in the recent revision of the species differentiation in Leucochloridiidae.

Although species belonging to the genus Leucochloridium are known to be common bird parasites worldwide [11-13], no species of parasites in the Leucochloridiidae has been reported among the wild bird populations in the Republic of Korea.

White's thrush, Zoothera aurea, is one of the 16 species of the family Turdidae recorded in Korea and belongs to the order Passeriformes [14]. White's thrush is a carnivorous bird that usually feeds on invertebrates including insects, their larvae, small slugs, and snails $[15,16]$. This bird is distributed throughout Korea and often reproduces in forests and parks, there is 
little research on this. Especially, there is no study on parasitic infestation of the Z. aurea.

In this study, for the first time, White's thrush was identified as a natural host of $U$. turdi in Korea. Therefore, we are going to confirm the characteristics of $U$. turdi by morphological and phylogenic analysis.

\section{MATERIALS AND METHODS}

\section{Sample collection and histological processing}

Six dead bodies of White's thrushes were dissected from January 2013 to August 2017 in the Daejeon city, Korea. These birds collected from the wildlife rescue center in Daejeon were immediately frozen after death and the flukes were subsequently recovered after thawing the bodies. One hundred and fifty-six flukes were harvested from the intestines of these birds. Twenty flukes were fixed with $10 \%$ formaldehyde without performing the flattening procedure for the light microscopic observation. The worms were stained with Semichon's acetocarmine, cleared with xylene, and mounted with Canada balsam. Additionally, to observe their internal organs, 5 flukes were embedded in paraffin, sectioned into slices of $4 \mu \mathrm{m}$, and then stained with hematoxylin and eosin. Measurements of the specimens are presented in micrometer as the range, with the mean in parentheses.

\section{Scanning electron microscopy (SEM)}

For the SEM examination, 5 parasites were washed 5 times with $0.2 \mathrm{M}$ cacodylate buffer ( $\mathrm{pH} 7.3$ ), fixed in $2.5 \%$ glutaraldehyde, and then post-fixed in $1 \%$ osmium tetroxide at $4^{\circ} \mathrm{C}$. The specimens were dehydrated in a graded ethyl alcohol series, dried with a critical point $\mathrm{CO}_{2}$ dryer, coated with gold and examined using SEM (S-4800, Hitachi) at $15 \mathrm{kV}$.

\section{Sequencing and phylogenetic analysis}

Twenty flukes were fixed in 100\% ethyl alcohol for the DNA sequencing. Genomic DNA was extracted from the collected parasites according to the manufacturer's instructions using a DNeasy ${ }^{\circledR}$ Blood \& Tissue Kit (Qiagen, Alameda, California, USA). Using 18S rRNA gene sequences of Leucochloridium, primers for common trematodes were designed using an online tool (Primer3Plus, http://www.bioinformatics.nl/cgi-bin/ primer3plus/primer3plus.cgi). The oligonucleotide sequences of primers were employed to detect the L. paradoxum $18 \mathrm{~S}$ rRNA gene (DNA) were 5'-AGGGAATGGGTGGATTTATT-3' (left primer) and 5'-AGACACGACTGAAAGGTTGC-3' (right primer). The primer sets were designed around 1,397 bp of the product, variable for all sequences aligned. Under standard conditions like $95^{\circ} \mathrm{C}$ for $30 \mathrm{sec}$, annealing at $60^{\circ} \mathrm{C}$ for $30 \mathrm{sec}$ and extension at $72^{\circ} \mathrm{C}$ for $1 \mathrm{~min}$, the polymerase chain reaction (PCR) was performed in a MyCycler Personal Thermal Cycler (Bio-Rad Laboratories, Hercules, California, USA) using the EmeraldAmp GT PCR Master Mix (Takara, Shiga, Japan) with a $1 \mu \mathrm{l}$ aliquot of DNA. Next, the PCR products were visualized via electrophoresis on a 1.2\% agarose gel and then purified using the QIAquick PCR Purification Kit (Qiagen). PCR amplicons were directly sequenced with the same primers as those used in PCR using the ABI Prism BigDye Terminator v.3.0 Ready Reaction Cycle Sequencing kits (Applied Biosystems, Foster City, California, USA). The sequencing data revealed around 726 bp of DNA. Furthermore, the sequence data were aligned using the Clustal Omega program (Clustal O 1.2.1). The phylogenetic tree was constructed based on the sequence analysis conducted using the neighbor-joining method by Basic Local Alignment Search Tool (BLAST) analysis. The neighbor-joining methods were based on a guide tree that provided pairwise and multiple alignment parameters [17].

\section{RESULTS}

\section{Morphological features of $U$. turdi}

Morphological characteristics observed in the collected specimens were as follows: the bodies were elongated, relatively wide with rounded extremities that were usually widest at the acetabular levels and 1,987-2,120 long by 819-831 wide $(2,009 \times 824)$ (Figs. 1, 3). Oral sucker subterminal, 351-432× 308-425 (402×391). Pharynx muscular, 142-179 ×78-170 $(163 \times 150)$. The prepharynx and esophagus were almost invisible. The ratio of the lengths of the oral sucker to that of the pharynx was 3.9:1 (Fig. 1A, C). The intestinal ceca were somewhat uniform in diameter throughout their entire length, each branch extending into the posterior end of the body to the area of the cirrus pouch (Figs. 1A, 2B). The ventral suckers were approximately 1.5 times larger than the oral suckers, occupying the central parts of the bodies and they were 536$673 \times 447-605(629 \times 553)$ in size (Figs. 1, 3A). Testes round or slightly elliptical, right testis $152-217 \times 114-172(183 \times 152)$, left testis $170-235 \times 99-145(205 \times 123)$ (Fig. 2). The cirrus pouches were somewhat spherical (Fig. 2B). The genital pores observed at the posterior ends of the bodies were median and 

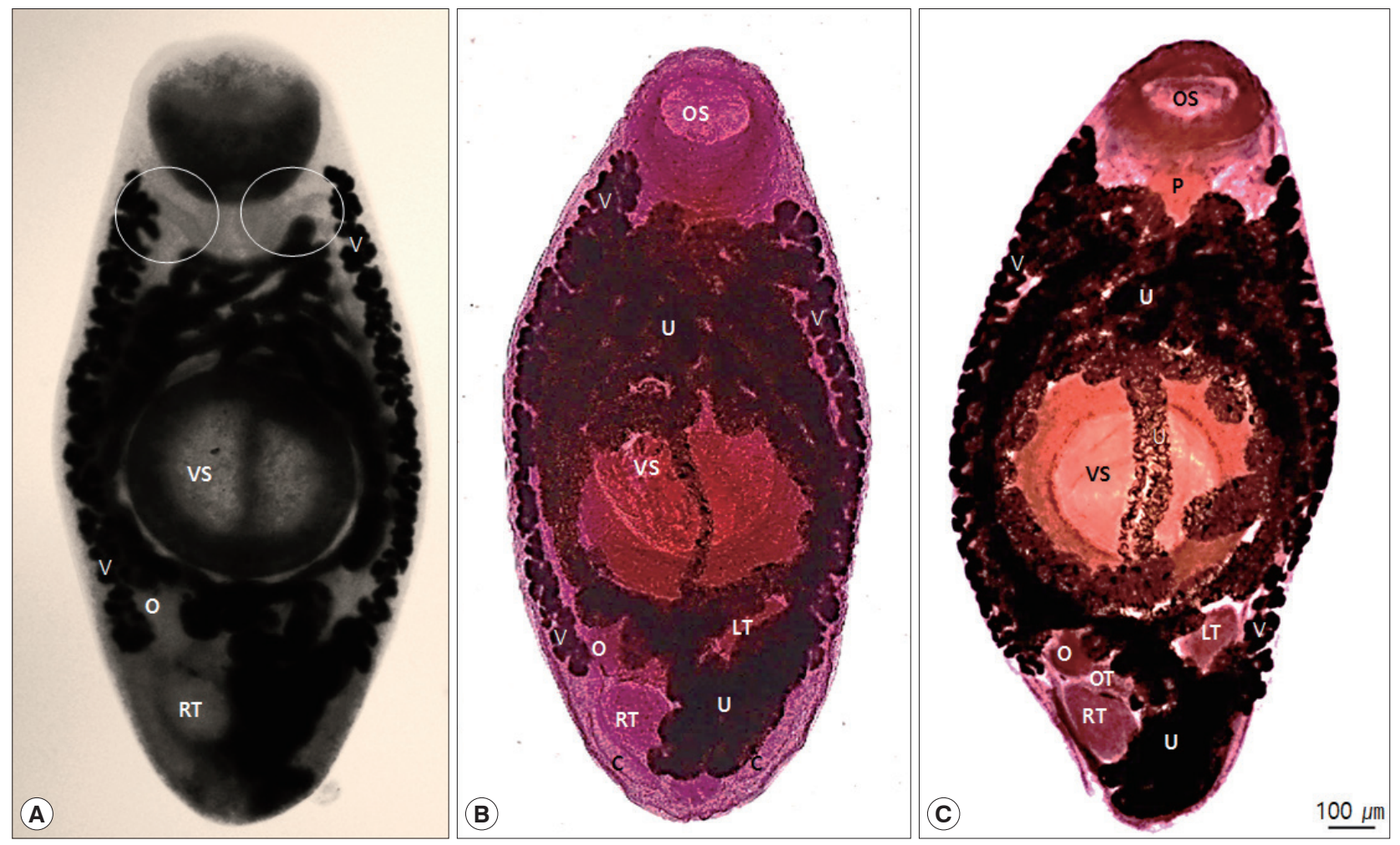

Fig. 1. Adult worm of U. turdi. (A) Unstained. The intestine ascends at level of pharynx before looping posteriorly (circles). (B and C) Adult worm of Stained with Semichon's aceto-carmine. U, uterus; LT, left testis; O, ovary; OS, oral sucker; OT, ootype; P, pharynx; RT, right testis; V, vitellaria; VS, ventral sucker. Vitellaria extend to level of right testis.
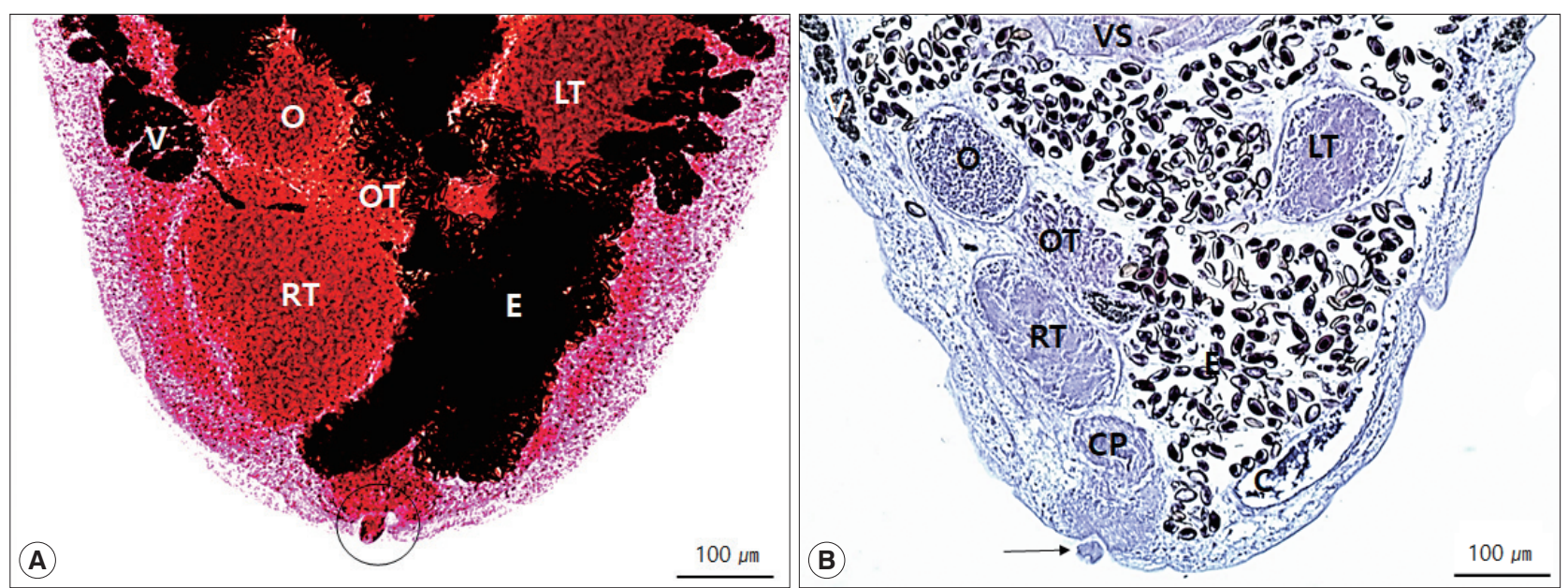

Fig. 2. Posterior part of U. turdi adult. (A) Stained with Semichon's aceto-carmine. (B) A cross sectioned posterior part stained with hematoxylin and eosin. (A) Note the cirrus (circle). (B) Posterior arrangement of genital organs. Arrow indicates cirrus.

ventro-terminal (Figs. 2, 3B). The reproductive glands occupied majority of the post-acetabular, intra-cecal areas and they were arranged in a triangle. The ovaries were spherical, 145$175 \times 111-135(161 \times 129)$ in size, situated behind the ventral suckers and lay symmetrically to the left testes (Fig. 2). Uterine seminal receptacles were present; ootypes were surrounded by the Mehlis' gland, present to the right of the ovaries and in the post-testicular regions. The Laurer's canals opened posteriorly towards the dorsal side (Fig. 3C, D). The vitellaria folliculars were found to begin adjacent to the levels of the posterior 

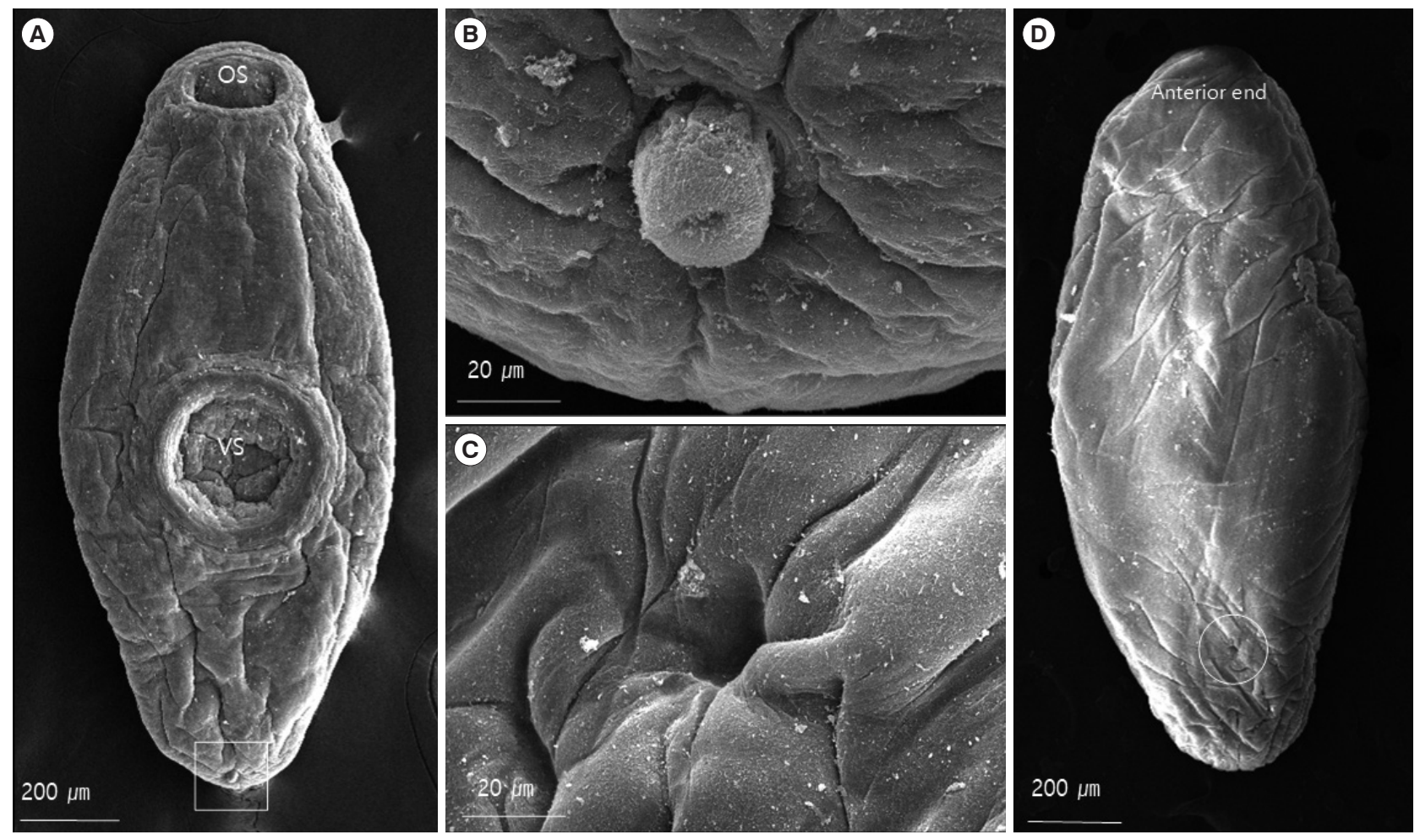

Fig. 3. Scanning electron micrographs of U. turdi. (A) Ventral view, evaginated cirrus (square). Note the size and location of the ventral sucker. (B) Higher magnification of evaginated cirrus. (C) Higher magnification of Laurer's canal. (D) Dorsal view, Laurer's canal (circle) is opened dorsally.

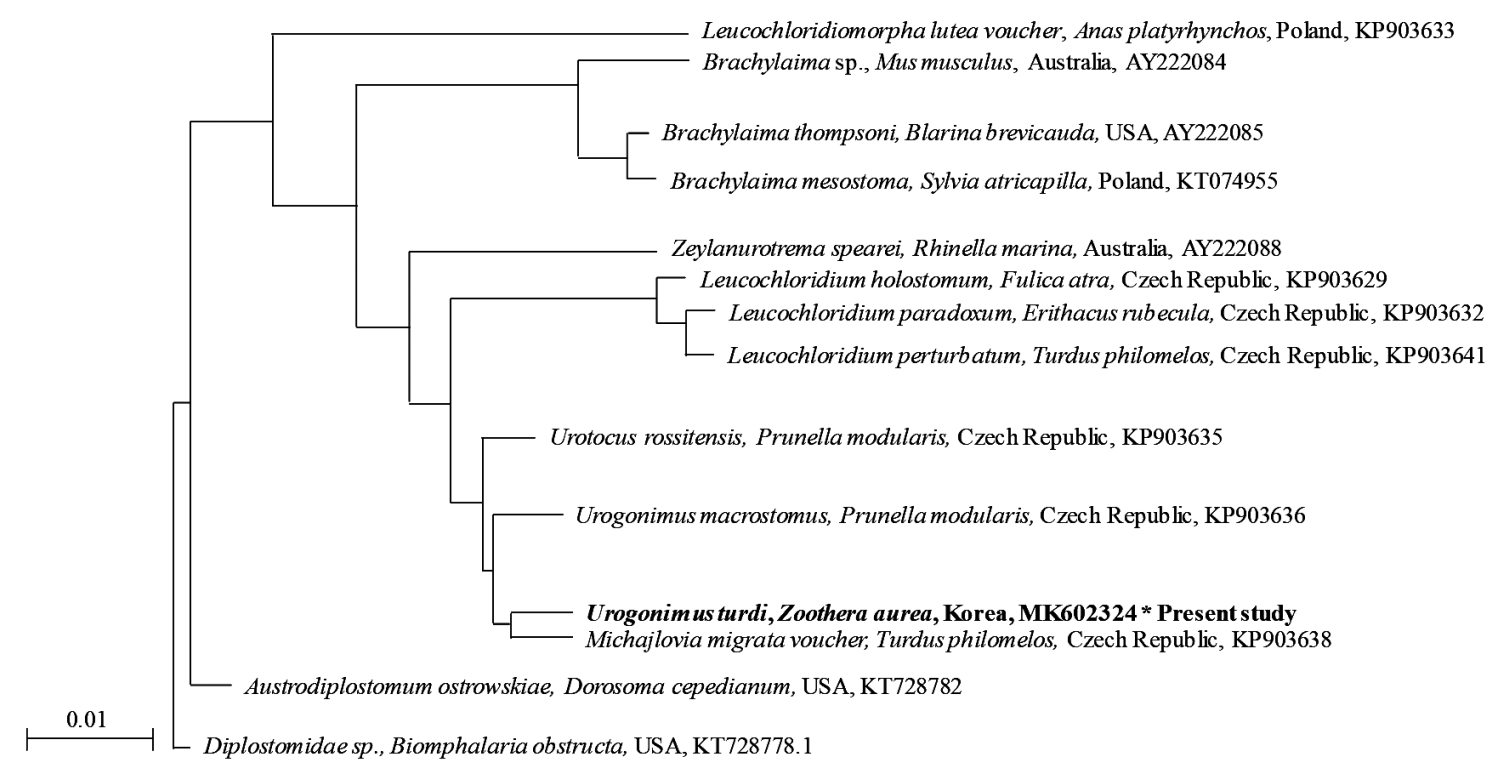

Fig. 4. Phylogenetic tree (Neighbor Joining) based on sequence analysis.

margins of the oral suckers and end at or near the levels of the right testes (Fig. 1). The uteri were filled with eggs and confined to the intercecal areas. The eggs were oval, embryonated, and 19.6-26.0 × 12.2-15.8 $(23.6 \times 14.6)$ in size.

In SEM study, the worms were found to have flat leaf-like bodies with prominent oral and ventral suckers (Fig. 3A). The 
bodies were covered with a smooth cytoplasmic layer and no tegumental spines were observed (Fig. 3D). The 2 suckers were remarkably well-developed. The ventral suckers were approximately 1.5 times larger than the oral suckers and located at the center of the bodies (Fig. 3A). The genital pores were situated at the median and ventro-terminal regions. The unspined cirri appeared from round to cylindrical (as the fully evaginated cirrus) in outline (Fig. 3B). The Laurer's canals opened on the dorsal surfaces of the bodies (Fig. 3C).

\section{Phylogenetic relationship}

To identify the genotype of the Urogonimus specimens in relation to their phylogenetic relationship with Leucochloridiida, the genomic DNA samples of the specimens were compared by phylogenetic analysis (Fig. 4). The partial sequence of the 18S rRNA in Urogonimus specimens was identified and compared to those of the parasites such as Leucochloridium holostomum (KP903629), L. paradoxum (KP903632), and L. perturbatum (KP903641). As a PCR amplicon, partial sequences (540 bp) of the Urogonimus specimens contained 15 variable sites of a common sequence which belonged to the clades of $L$. holostomum, L. paradoxum, and $L$. perturbatum, respectively. The partial sequences of the 18S rRNA gene of the Urogonimus specimens showed high similarity with those of the parasites $L$. holostomum (97.6\%), L. paradoxum (97.6\%), L. perturbatum (97.4\%), Michajlovia migrata (99.3\%), Urotocus rossitensis (99.3\%), and U. macrostomus (98.5\%). The results of the phylogenetic analysis validate the fact that these samples belonged to the same clades as Urogonimus based on their key morphological features. After the sequence of Urogonimus specimens was compared with several genes, the sequence data was inferred using the neighbor-joining method (Fig. 4). The sequence of Urogonimus specimens was also identified to be of $U$. turdi, which is genetically close to M. migrata $(99.3 \%), U$. rossitensis (99.3\%), and U. macrostomus (98.5\%). Although the morphology of $U$. turdi was also found to match with the above Leucochloridium sp., the identification strongly supported the fact that the samples are closer to the clades of the Urogonimus species. Finally, we submitted the sequence data to GenBank and received the GenBank accession number for our nucleotide sequence (BankIt MK602324).

\section{DISCUSSION}

Despite recent researches on Leucochloridiidae, there has been a great confusion regarding their genera, species, synonyms, nomenclature, and systematic position [1,10,18-20]. Currently, the Leucochloridiidae consists of the 3 genera Urotocus Looss, 1899, Urogonimus Monticelli, 1888, and Leucochloridium Carus, 1835. These 3 genera can be distinguished based on the proportions of their suckers, position of their ventral sucker, arrangement of their gonads, position of their genital pores, and course of the uterus. Michajlovia Pojmanska, 1973 was included in Leucochloridiidae by Pojmanska (1973) and is now regarded as Incertae sedis [21]. The morphological characteristics of the genus Urotocus among all the 3 genera include a long body and a sucker that is relatively poorly developed. On the othr hand, the general shape of the other 2 genera are close to ellipsoidal and have well-developed suckers. The species belonging to the genera Leucochloridium and Urogonimus are very similar, and hence, difficult to distinguish from one another, but there are some morphological differences in the location of their genital pores, presence of a spine on the surface of their bodies and distribution of the vitelline glands. In the species belonging to the genus Urogonimus, the genital pores are often at the posterior end of the body and vitelline glands extend from the ceca to the region of the oral suckers and that of the gonads, but not reaching cecal extremities, and have no spines on their body surfaces. In the species belonging to the genus Leucochloridium, the genital pore is distinctly at the dorsal region and the vitelline glands extending from the posterior to the cecal extremities or may stretch even further posteriorly and have fine spines on their body surfaces [21].

In the genus Urogonimus, the vitellaria are relatively short and extend only to the level of the right testis. In a recent study, among the species of this genus, unique morphological features were observed in $U$. turdi; the genital glands were arranged in a triangle at the posterior region of the body and the acetabulum was slightly post-equatorial and this was a unique phenomenon in $U$. turdi when compared to the other species of Urogonimus [10]. In the present study, the vitellaria of the fluke taken from White's thrush extend from the level of the oral sucker to that of the right testis and the position of the genital glands and the acetabulum is very similar to that of $U$. turdi reported in the previous study [10]. Moreover, our specimens are very similar to those of $L$. turdi obtained from the cloaca of $L$. cardis from Kutama near Kyoto, Japan in 1937, as described by Yamaguti [8]. It has been noticed that the size of the acetabulum was $0.34-0.68 \mathrm{~mm}$ in diameter which was considerably larger than the oral suckers $(0.22-0.48 \mathrm{~mm}$ in di- 
ameter). In the present study, the acetabulum is approximately 1.5 times larger than the oral sucker.

In 1979, Iskova [22] reported an infection by $U$. turdi in Ukrainian passerine birds and Ching [3] also reported an infection of $L$. turdi in varied thrushes found in British Columbia. Iwaki et al. [9] suspected that the acetabulum of $U$. turdi that infected Ukrainian passerine birds was U. macrostomus because it was almost in the equator. The size of $L$. turdi collected from the varied thrushes in British Columbia was 1,637×639 $\mu \mathrm{m}$ and it is much smaller than the fluke $(2,009 \times 824 \mu \mathrm{m})$ described in the present study. Accordingly, it is unclear that the fluke in British Columbia would be identified as L. turdi because of the difference in size. Although much uncertainty still exists in distinguishing between the species of the genera Leucochloridium and Urogonimus, the specimens collected from the Korean White's thrushes can be identified as those belonging to the genus Urogonimus based on the key provided by Kagan [10].

Additionally, the nuclear ribosomal DNA, the 18S rRNA gene, was used to study the inter- and intraspecific relationships, since it is highly repeated and contains variable regions flanked by more conserved regions $[23,24]$. Although the genetic divergence of $U$. turdi suggests its conspecific relationship with other Leucochloridium sp., the 18S rRNA sequences proved to be useful in distinguishing $U$. turdi from other parasites such as L. holostomum (KP903629), L. paradoxum (KР903632), and $L$. perturbatum (KP903641). In conclusion, we can determine that our specimen has been validated as $U$. turdi, and this is the first identified species of $U$. turdi from $Z$. aurea in Korea.

\section{ACKNOWLEDGMENTS}

This research was supported by Basic Science Research Program through the National Research Foundation of Korea (NRF) funded by the Ministry of Education (Grant No. 2017RIDIAIB06031728).

\section{CONFLICT OF INTEREST}

The authors declare no conflict of interest related to this study.

\section{REFERENCES}

1. Bakke TA. A revision of the family Leucochloridiidae Poche (Digenea) and studies on the morphology of Leucochloridium para- doxum Carus, 1835. Syst Parasitol 1980; 1: 189-202.

2. Locke SA, Lapierre AR, Byers K, Proctor H, McLaughlin JD, Marcogliese DJ. Molecular and morphological evidence for the Holarctic distribution of Urogonimus macrostomus (Rudolphi, 1803) Monticelli, 1888 (Digenea; Leucochloridiidae). J Parasitol 2012; 98: 880-882.

3. Ching HL. Helminths of varied thrushes, Ixoreus naevius, and robins, Turdus migratorius, from Britisg Columbia. J Helminthol Soc Wash 1993; 60: 239-242.

4. Fischthal JH, Kuntz RE. Brachylaimid and Dicrocoeliid trematodes of birds from Palawan Island, Philippines. Proc Helminthol Soc Wash 1973; 41: 94-104.

5. Heneberg P, Sitko J, Bizos J. Molecular and comparative morphological analysis of central European parasitic flatworms of the superfamily Brachylaimoidea Allison, 1943 (Trematoda: Plagiorchiida). Parasitology 2016; 143: 455-474.

6. Ishii N. Studies on bird trematodes I. (1) Observations on trematode species. (II) Detection of new species. Jpn Exp Med J 1932; 16: 1205-1231 (in Japanese).

7. Yamaguti S. Part 5. Trematodes of birds III. Jpn J Zool 1935; 6: 159-182.

8. Yamaguti S. Studies on the helminth fauna of Japan. Part 25. Trematodes of birds IV. Jpn J Zool 1939; 8: 129-210.

9. Iwaki T, Okamoto M, Nakamori J. Urogonimus macrostomus (Digenea: Leucochloridiidae) from the rustic bunting, Emberiza rustica, in Japan. Parasitol Int 2009; 58: 303-305.

10. Kagan IG. Revision of the subfamily Leucochloridiinae Poche, 1907 (Trematoda: Brachylaemidae). Am Midl Nat 1952; 48 257-301

11. Casey SP, Bakke TA, Harris PD, Cable J. Use of ITS rDNA for discrimination of European green- and brown-banded sporocysts within the genus Leucochloridium Carus, 1835 (Digenea: Leucochloriddidae). Syst Parasitol 2003; 56: 163-168.

12. Kharoo VK. A new species of the genus Leucochloridium carus, 1835 (Trematoda: Leucochloridiidae (Poche, 1907) Dollfus, 1934 from the spotted red shank Totanus fuscus from Allahabad, India. Indian J Fund Life Sci 2012; 2: 276-280.

13. Rzad I, Hofsoe P, Panicz R, Nowakowski JK. Morphological and molecular characterization of adult worms of Leucochloridium paradoxum Carus, 1835 and L. perturbatum pojmanska, 1969 (Digenea: Leucochloridiidae) from the great tit, Parus major L. 1758 and similarity with the sporocysts stages. J Helminthol 2014; 88: 506-510.

14. Han SW, Lee DP. A Comparative study of the characteristics of nest sites among three species of turdidae. Korean J Orni 2015; 22: $13-19$.

15. Park JG, Seo JH. A Photographic Guide to the Birds of Korea. Seoul, Korea. Shingubook. 2008, pp 1-515 (in Korean).

16. Won BO. Illustrated Encyclopedia of Fauna \& Flora of Korea. Vol. 25, Animal. Seoul, Korea. Ministry of Education. 1981, pp 876-886 (in Korean).

17. Hong EJ, Sim C, Chae JS, Kim HC, Park J, Choi KS, Yu DH, Yoo JG, Park BK. A horsehair worm, Gordius sp. (Nematomorpha: 
Gordiida), passed in a canine feces. Korean J Parasitol 2015; 53: 719-724.

18. Pojmanska T. Michajlovia migrata gen. n., sp. n. (Trematoda, Brachylamata)-the morphology of the adult. Acta Parasitol Pol 1973; 21: 9-20.

19. Pojmanska T. Family Leucochloridiidae Poche, 1907. In Gibson DI, Jones A, Bray RA eds, Keys to the Trematode, Vol. 1. London, UK. CABI Publishing and Natural History Museum. 2002, pp 47-51.

20. Bakke TA. Shape, size, and surface topography of genital organs of Urogonimus macrostomus (Rudolphi, 1803) Monticelli, 1888 (Digenea), revealed by light and scanning electron microscopy. Z Parasitenk 1977; 54: 299-307.
21. Gibson DI, Jones A, Bray RA. Keys to the Trematoda, Vol. 1. CABI Publishing and Natural History Museum, London, UK. 2002, pp 31-51.

22. Iskova NI. Trematodes of the family Leucochloridiidae from passerine birds of the Ukraine. Vestn Zool 1979; 5: 46-55 (in Russian).

23. Hillis DM, Dixon MT. Ribosomal DNA: molecular evolution and phylogenetic inference. Q Rev Biol 1991; 66: 411-453.

24. Morgan JAT, Blair D. Nuclear rDNA ITS sequence variation in the trematode genus Echinostoma: an aid to establishing relationships within the 37-collar spine group. Parasitology 1995; 111: 609-615. 
